\title{
PROVISIONING CONTEXT-AWARE ADVERTISEMENTS TO WIRELESS MOBILE USERS
}

\author{
Qusay H. Mahmoud \\ Department of Computing and Information Science \\ University of Guelph, Guelph, ON, N1G 2W1 Canada \\ qmahmoud@cis.uoguelph.ca
}

\begin{abstract}
Mobile advertising, which is an area of mobile commerce, is a form of advertising that targets users of handheld wireless devices such as mobile phones and personal digital assistants (PDAs). Given the constraints of such devices, mobile advertisements should be context-aware in the sense that the behaviour of such value-added services is mostly driven by information based on user's location, time, user preferences, and the task at hand. This paper presents the design and prototyping of a system for provisioning context-aware advertisements to wireless mobile users. We use mobile agents because such autonomous entities have characteristics that can benefit mobile devices and the wireless environment. We have constructed a proof of concept implementation using Java technologies with support for WAP-enabled and J2MEenabled devices.
\end{abstract}

\section{INTRODUCTION}

For many websites advertising is the major source of revenue. Yet, if we look at the forms of advertisements used on the web, one will see that the majority of them are using serendipitous advertising - a form of advertising where every user is deemed identical and is thus shown the same advertisements. Too often, we have seen advertisement banners on websites selling products we will never buy, or are giving away promotions to only patrons of far-off countries. As more and more websites enable users of handheld devices to browse their content, such websites need to devise new approaches for provisioning tailored advertisements to the user's location, preferences, and the task at hand. Mobile advertising, which is an area of mobile commerce, is a form of advertising that targets users of handheld wireless devices such as mobile phones and PDAs. In Comparison with traditional advertising, the main advantages of mobile advertising are: personal touch, tailor-made to suit individuals, and hence can be considered cost effective. Mobile advertising can reach the target customers anywhere anytime; customers can be aware of mobile advertisements and services while they are walking. In order to promote the selling of products or services, all the activities required to communicate with the customers are transferred through mobile devices. Combining with the customer's user profile and context situation, advertising companies can provide the target customers exactly the advertisement information they desire, not just "spam" them with advertisements they are not interested in.

The devices on which these value-added services are pushed onto or downloaded to operate in an environment that imposes constrains such as: wireless network environments are unreliable and bandwidth is low, and the very mobility of devices increases the risk that a connection will be lost or degraded. More importantly, mobile services must work within the daunting constraints of the devices themselves, which include: memory, processing power, input capabilities, and size of display. The display might be as small as 96 pixels wide by 54 pixels high and 1 bit deep (black and white), and therefore the amount of information that can be squeezed into such a tight screen is severely limited. It is therefore important that mobile advertisements take into account the user's context, optimize resource usage, and minimize input effort imposed on the user.

We believe that by using the information retrieval and filtering capabilities of mobile agents and location information about the user, there exists a good opportunity for value-added services to be provided to the end-users. This also brings about a new way for cellular phone service providers to achieve competitive advantage by competing not only on the basis of price and packaging, but also on the basis of the set of value-added services that they provide to their clients. In order to overcome the input/output limitations brought about by mobile devices, the system should be free of user's intervention. To that end, we propose to use mobile agents to provisioning context-aware advertisements to mobile users. Schilit and Theimer first introduced the concept of context-awareness in the project Active Map [12] in which they took advantage of the location concept to define the context as people, object, and the changes that occur to them. Dey and Abowd [13] gave the following definition which we follow in the design of our system: "Context is any information that can be used to characterize the situation 
of an entity. An entity is a person, place or object that is considered relevant to the interaction between a user and an application, including the user and applications themselves". In addition, they stated that a system is context-aware if it uses context to provide relevant information and/or services to the user, where relevancy depends on user's task.

The rest of this paper is organized as follows. Section 2 provides an overview of mobile agents and discusses the benefits of using them in mobile devices and the wireless environment. Mobile agents in our system are used for context filtering and searching. Section 3 presents the proposed system architecture, and discusses how the system achieves context-awareness. The implementation of the prototype is presented in Section 4. Section 5 discusses the related work. Finally, conclusions and future work are presented in Section 6.

\section{MOBILE AGENTS}

The characteristics of mobile devices and the wireless environment call for an architecture that reduces the load on the wireless link and supports disconnected operations. We believe that mobile agents are suitable to be used in a wireless environment for provisioning services and mobile advertisements. A mobile software agent is software entity that can move in a heterogeneous network to carry out various tasks on behalf of its user. It carries its actions in an autonomous, active, and reactive manner. Mobile agents have several advantages for mobile services $[2,3]$. Mobile agents run autonomously and asynchronously; they can adapt dynamically according to the execution environment; they can reduce network traffic; and they can be packaged with the specific tasks and sent to the destination host. When they arrive at their destination, they can carry out the tasks locally.

There are two ways to employ software agents in handheld devices: (1) have an agent platform run on the device and therefore allow agents to run on the device; or (2) allow devices to access and use remote agents running on wired networks. We believe that the two approaches are viable and each has its own advantages and disadvantages, as well as its own domain specific applications. Using mobile agents in the real world involves solving many non-trivial problems. The main challenges can be categorized generally into (1) what is the best way to deploy mobile agents in a wireless environment, (2) security concerns, (3) user resistance, and (4) that of a lack of an easy integration with the existing web infrastructure. More information on this can be found in [9]. It is important to note that in the proposed system software agents run on a remote server hosted by an Internet service provider or a cellular network service provider. In other words, agents do not run on users' handheld devices. The advantages of this are: (1) the user is saved from the headaches of software installs and updates; and (2) the complexity of software agents is hidden away from the end-users.

\section{CONTEXT-AWARE ADVERTISING}

The aim of our approach is to design a system for provisioning context-aware mobile advertisements to mobile users. The system is built on top of an earlier system we have designed for personalizing services [8] as well as our work on using mobile agents for locationaware advertising [6]. We have added support for defining advertisements and provisioning them. A high-level architecture of our system is shown in Figure 1. There are three main parts in this architecture: the clients, the Entry Server, and the Service Server. The Entry Server is responsible for the interaction between devices and the rest of the system. The Service Server hosts advertisements, services and service descriptions, and allows agents that migrate from the Entry Server to run there and select services and advertisements. Note that the advertisements and services themselves might be located on a different server. The system is capable of handling J2ME and WAP devices. In order to support WAP devices, a WAP Gateway is used on the Entry Server side that can transfer requests and services between WAP devices and the Entry Server. The functionality of the components is described in details in [8].

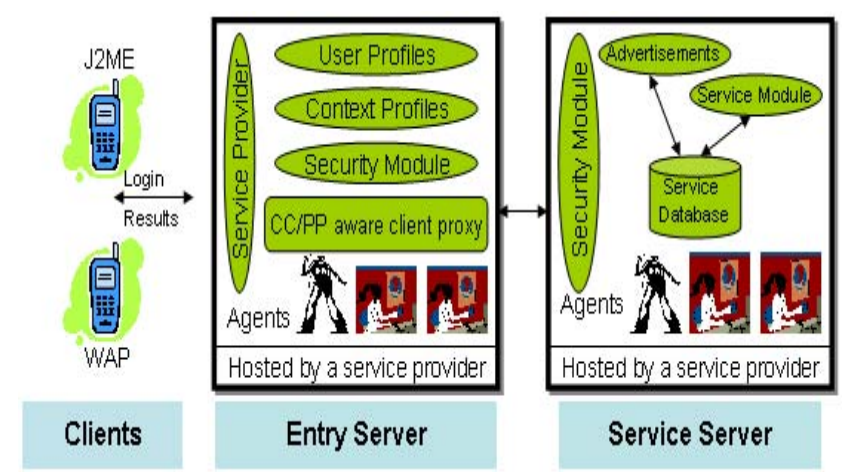

Figure 1. Architecture of the proposed system.

The benefits of the proposed system are: (1) it enables the provisioning of context-aware value-added services (such as context-aware advertisements) to WAP and J2ME devices; (2) it overcomes low bandwidth and wireless network disconnection, and this is achieved by reducing the communications over the wireless link between the device and the Entry Server. This communication is further reduced because (3) the system is able to operate without user's intervention. The input is injected by the Entry Server based on the user's profile; and (4) the system architecture is not tied to any agent platform.

\subsection{Context-Awareness}

We use location, time, and user preferences and device capabilities for defining the context as follows: 
Location: In a mobile computing environment, location information is an important aspect for a context-aware system. There are two reasons for it. Firstly, after a mobile user sends a request, she may move to another place or turn off her device. When the system gets the results and is ready to send them to the user, the location of the user may have changed and thus his or her interest in the results may no longer be the same. Secondly, some query results may include several items; for example, all restaurants in a city. But if the user just needs localized information, such as restaurant locations within a radius of one mile, the system should know where the user is and just provide information relevant to the user. Due to these two reasons, we use a Context Profile Module in the Entry Server to track user's location in system.

Time: This contextual information is not difficult to obtain, and the system makes use of the built-in clock of the server to retrieve it. For example, when a user searches for restaurant information at lunch time, the system needs to compare the query time with the opening time of restaurants for making the final recommendation. In this situation, the system needs to know the current time.

User Preferences and Device Capabilities: This information can be obtained from CC/PP profiles [1]. The Service Provider can get the mobile user's preferences and device capabilities easily. On the client-side, the mobile device sends reference profiles and profile differences through some protocols such as W-HTTP or CC/PP-ex.

\subsection{Provisioning Value-Added Services}

In order to provision new as well as existing value-added services, we employ a Service-Oriented Architecture (SOA), which is an architectural style for building software applications that use services available in a network such as the Web. It promotes loose coupling between software components so that they can be reused. SOA uses the find-bind-execute paradigm. Adapting this paradigm, content and value-added service providers register their services in a public registry. This registry is used by our system to find value-added services that match certain criteria. If the registry has such a service, it provides our system with a contract and an endpoint address for that service.

Service and content providers register their products information through interfaces provided by Service Module, and thus do not have direct contact with the customers. In addition, it is important to note that customers must agree to accept mobile advertisements when they subscribe to value-added services; however, customers have control on how and when to receive advertisements, so they can turn off that functionality or indicate not to receive advertisements at certain times. The Entry Server uses agents to filter context based on the user's profile and context profile, and dispatches mobile agents to search for these mobile advertisements information. When agents return to Entry Server along with the searching results, Service Provider selects and pushes the (already relevant) mobile advertisements information to the potential customers according. There are two types of advertisements here, advertisements that can be pushed along with the results of a product search as outlined in our previous work in [6], or they can be treated as value-added services that mobile users subscribe to (e.g. send promotions to my mobile device as I enter a shopping mall). As an example of a value-added service, consider a user who will be going to the mall on a Saturday, and before they leave home (or while on the way) they login into the system and enter products they are interested in buying today. The system receives the request and dispatches agents to the service provider to find suitable products and services the user might be interested in, taking into account the users preferences, device capabilities, and of course the location is already known in this case (the mall). As the user enters the mall, the device starts receiving information of products and services relevant to the user. In addition, there will be advertisements from other vendors in the mall.

\section{PROTOTYPE IMPLEMENTATION}

A proof of concept implementation has been constructed using Java technologies. The agent platform is implemented using Java Remote Method Invocation (RMI). In the Entry Server, the Service Provider is implemented using Servlets, and it is in charge of communicating with mobile users and the WAP Gateway. When a customer's service request is received, the Service Provider creates the customer's user profile through JSR 188 API (for CC/PP processing) and stores it in User Profile Module. Customers can be tracked at a service point using wireless technologies, so a number of Entry Servers were provided as access points in service areas. When a mobile customer logs into the system, her device will try to connect to the nearest Entry Server. The Entry Server detects the customer's context, such as location, and stores them in the Context Profile Module. In order to manage the user profile and context profile, MySQL is used in the Entry Server. The security module is implemented using Java Cryptographic Extension (JCE).

For J2ME customers, we use J2ME MIDP 2.0 Push Registry to push mobile advertisements information. The push registry is part of the application management system (AMS), the software in the device that's responsible for each application's life-cycle (installation, activation, execution, and removal). Once installed on a mobile device, a MIDlet can be activated in one of the three ways: user request, incoming network connection, and scheduled alarm. The push registry is in charge of the last two ways, so it can enable MIDlets to set themselves up to be launched automatically. To become push-enabled, MIDlets must register with the push registry using static registration or dynamic registration; hence customers must indicate that they wish to accept mobile advertisements. 
For WAP customers, Service Provider pushes the selected mobile Advertisements to them through WAP push technology. The Push Initiator (PI) can transmit Push content and delivery instructions to a Push Proxy Gateway (PPG), which then delivers Push content to the WAP client according to the delivery instructions. The PI is typically an application that runs on a Web Server, and Service Provider fulfills the function of PI in our system. It communicates with PPG using the Push Access Protocol (PAP) over HTTP. The PPG uses the Push Over-The-Air (OTA) Protocol to deliver the Push content to the client. This was implemented by using the Nokia Toolkit 4.0 Starter Package, and Nokia WAP Gateway Simulator 4.0 is used as PPG in our system.

\section{RELATED WORK}

Several research papers list mobile advertising as one of the future possibilities of mobile commerce. For example, Varshney and Vetter [4] suggested that mobile advertising will be a very important class of mobile commerce. They augmented location information with the personalization of the delivery by obtaining the history of the user's purchases or consulting the user at an earlier stage. Barnes [5] introduced the concept of tempting nearby users into the stores and delivering location messaging related to security in a particular area of the city, for example. Ranganathan and Campbell [7] discussed mobile advertising in the context of pervasive computing environments; they presented a list of challenges and some ideas for solutions for advertising in pervasive environments. Randell and Muller [10] presented the Shopping Jacket infrastructure that uses GPS and local pingers in stores for positioning. Wearers are alerted when passing an interesting shop. Finally, Aalto et al [11] introduced B-MAD (Bluetooth Mobile Advertising), a location-aware mobile advertising system based on WAP Push and Bluetooth positioning technology that is capable of delivering permission-based location-aware mobile advertisements to mobile phones.

The novel contribution of our approach is the design and implementation of a system capable of provisioning advertisements to wireless mobile users; the system uses the CC/PP to gather context information about users, and mobile agents as a context filter and searching mechanisms. A business model such as the one we have previously proposed in [9] can be applied in the proposed system to allow businesses to form partnerships to solve the usability and security problems of mobile agents.

\section{CONCLUSION AND FUTURE WORK}

This paper presented an agent-based system for provision context-aware advertisements to wireless mobile users of J2ME and WAP devices. We discussed the architecture of the proposed system and the proof of concept implementation we have built using Java technologies. This is a novel approach since it differs from all the related work in three important areas: (1) the user preferences and device capabilities which are used in context filtering are based on the W3C standardized profile (CC/PP); (2) the system supports two widely used technologies for developing wireless applications, namely WAP and J2ME; and (3) the advertising infrastructure is based on SOA, where mobile agents are used for context filtering. For future work, we plan to add more valueadded services, enhance the functionality of the system, and evaluate its performance in a live wireless environment. In addition, we plan to address the privacy concerns from the perspective of customers.

\section{REFERENCES}

[1] W3C CC/PP Information Page, www.w3.org/Mobile/CCPP. [2] D.B. Lang and M. Oshima, "Seven Good Reasons for Mobile Agents," Comm. of the ACM, Vol. 42, No. 3, pp. 88-89, 1999.

[3] J. Claessens, B. Preneel, and J. Vandewalle, "How Can Mobile Agents Do Secure Electronic Transactions on Untrusted Hosts? A Survey of Security Issues and the Current Solutions," ACM Transactions on Internet Technology, Vol. 3, No. 1, pp 2848, February 2003.

[4] U. Varshney, and R. Vetter, "Mobile Commerce: Framework, Applications and Networking Support". In Mobile Networks and Applications, Vol. 7, No. 3, 185-198, 2002.

[5] S. J., Barnes, "Known by the Network: The Emergence of Location-Based Mobile Commerce". In Advances in Mobile Commerce Technologies, 171-189, 2003.

[6] Q. H. Mahmoud and L. Yu, "Mobile Agents for LocationAware Advertising in Mobile Wireless Environments," in Proc. of the Tenth Americas Conference on Information Systems, New York, New York, USA, pp. 2168-2175, 2004.

[7] A. Ranganathan, and R.H. Campbell, "Advertising in a Pervasive Computing Environment", in Proc. of the $2^{\text {nd }}$ international workshop on Mobile commerce, Atlanta, Georgia, USA, pp. 10-14, 2002

[8] Q.H. Mahmoud, and Z. Wang, "Customizing and Delivering Mobile Services using Software Agents and CC/PP". In Proceedings of the IEEE Consumer Communications and Networking Conferences (CCNC2006), Las Vegas, Nevada, USA, pp. 1114-1118, 2006.

[9] Q.H. Mahmoud, and L. Yu, "An Architecture and Business Model for Making Software Agents Commercially Viable", Proceedings of the 38 Hawaii International Conference on System Sciences (HICSS-38), Big Island, Hawaii, USA, 2005.

[10] C. Randell, and H. Muller, "The Shopping Jacket: Wearable Computing for the Consumer". In Personal and Ubiquitous Computing, Vol. 4, No. 4, 241-244, 2000.

[11] L. Aalto, N. Gothlin, J. Korhonen, and T. Ojala, "Bluetooth and WAP Push Based Location-Aware Mobile Advertising System," MobiSYS'04, Boston, Mass., USA, pp. 49-58, 2004.

[12] B. Schilit, and M. Theimer, "Disseminating Active Map Information to Mobile Hosts", IEEE Network, Vol. 8, No. 5, pp. 22-32, 1994.

[13] A.K. Dey, and G.D. Abowd, "Towards a Better Understanding of Context and Context-Awareness", in the Workshop on The What, Who, Where, When, and How of Context-Awareness, as part of the 2000 Conference on Human Factors in Computing Systems, The Hague, The Netherlands, pp. 304-307, 2000. 